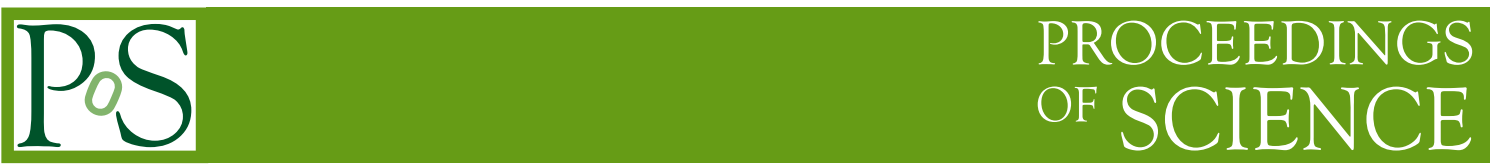

\title{
Jets, Winds, and Accretion in T Tauri Stars
}

\author{
B. Nisini* \\ INAF-Osservatorio Astronomico di Roma \\ E-mail: brunella.nisinieinaf.it
}

Forbidden lines emission in T Tauri stars is a powerful probe of the presence of high velocity jets and slow winds originating from the inner region of the circumstellar disc. Such phenomena are strictly linked to mass accretion, contributing to the disc evolution and dispersal. We summarize here the results of a survey on the [OI] $6300 \AA$ line emission in $131 \mathrm{~T}$ Tauri stars of nearby star forming regions, observed with the X-shooter spectrograph at the Very Large Telescope (VLT). The line profile was deconvolved into a low velocity component $\left(\mathrm{LVC},\left|\mathrm{V}_{r}\right|<40 \mathrm{~km} \mathrm{~s}^{-1}\right)$ and a high velocity component $\left(\mathrm{HVC},\left|\mathrm{V}_{r}\right|>40 \mathrm{~km} \mathrm{~s}^{-1}\right)$, originating from slow winds and high velocity jets, respectively. We found a strong correlation between the [OI]6300 $\AA$ luminosity of both the LVC and HVC, and the stellar and accretion parameters of the central sources, with similar slopes for the two components. Mass ejection rates $\left(\dot{M}_{j e t}\right)$ measured from the HVC [OI]6300Å line luminosity span from $\sim 10^{-13}$ to $\sim 10^{-7} \mathrm{M}_{\odot} \mathrm{yr}^{-1}$, and the corresponding $\dot{M}_{\text {jet }} / \dot{M}_{\text {acc }}$ ratio ranges from $\sim 0.01$ to $\sim 0.5$, with an average value of 0.07 . However, considering the upper limits on the non-detected HVC, we infer a $\dot{M}_{j e t} / \dot{M}_{a c c}$ ratio $<0.03$ in more than $40 \%$ of sources. We argue that most of these sources might lack the physical conditions needed for an efficient magnetocentrifugal acceleration in the star-disc interaction region.

Accretion Processes in Cosmic Sources - II - APCS2018

3-8 September 2018

Saint Petersburg, Russian Federation

${ }^{*}$ Speaker. 


\section{Introduction}

High velocity jets are a common phenomenon during the active phase of accretion in Young Stellar Objects (YSOs), as they are able to efficiently extract angular momentum accumulated in the disc, allowing disc matter to accrete onto the star (e.g. [1] ). However, the frequency of the jet phenomenon among the general population of YSOs, and its link with the disc mass accretion, is still far from being settled. The question on how common jets are in active accreting stars has a major relevance in the context of disc dispersal, as jets can significantly modify the structure of the disc region involved in the launching, impacting its evolution and the formation of planetary systems. A direct way to infer the presence of jets and winds in CTT stars is through observations of atomic forbidden lines (e.g. [2,3,5]), and of the [OI]6300 transition in particular, being the brightest among the optical lines. The [OI]6300 $\AA$ line commonly exhibits two distinct components: a Low Velocity Component (LVC), peaking at velocities close to systemic one or only slightly blue-shifted, and a High Velocity Component (HVC) observed at velocities up to $\sim \pm$ $200 \mathrm{~km} \mathrm{~s}^{-1}$. The HVC is directly connected with the extended collimated jets, while the origin of the LVC is still unclear. It comes from a compact and denser region with respect to the HVC $([2,3])$ and it is usually associated to an atomic photo-evaporated or magneto-hydro-dinamic (MHD) slowwind (e.g. [6,7]). In this contribution, we summarise the results obtained with a statistical study on the $[\mathrm{OI}] 6300 \AA$ emission in a sample of $131 \mathrm{~T}$ Tauri stars in the Lupus, Chamaeleon and $\sigma$ Orionis star-forming regions ([4]) observed with the X-shooter instrument at the VLT. These observations are part of a project aimed at characterizing the population of young stellar objects with discs in nearby star-forming regions ([8,9]). As part of this project, the stellar and accretion properties of the sample have been measured in a uniform and self-consistent way, providing a unique database where the properties of the $[\mathrm{OI}] 6300 \AA$ emission can be analysed and correlated with the other derived parameters in a homogeneous fashion .

\section{The sample and $[\mathrm{OI}] 6300 \AA$ decomposition}

The adopted sample consists of 131 Young Stellar Objects (YSOs) observed with the Xshooter instrument. In particular, the sample includes sources in the Lupus (82 sources), Chamaeleon (41 sources) and $\sigma$ Orionis (8 sources) star forming regions, whose stellar and accretion properties have been already analysed in previous papers ( [10, 8] for Lup; [11] for $\sigma$ Ori; [9] for Cha). We focus our analysis on the $[\mathrm{OI}] 6300 \AA$ line which is the brightest among the forbidden lines in the spectral range of our data . As discussed in the introduction, the [OI] $6300 \AA$ profile can be decomposed in a LVC, close to systemic velocity, and a blue- or red-shifted HVC. To separately derive line center and width of the different components we fitted the [OI] line profile with one or more gaussians (see Fig. 1). We define the velocity that separates the LVC from the HVC as $40 \mathrm{~km} \mathrm{~s}^{-1}$, which roughly corresponds to the resolution attained for most of our objects. Line fluxes have been then converted into intrinsic luminosities adopting the $A_{V}$ derived from the stellar parameters fitting procedure (see e.g. [10]).

\section{Statistics and correlation with stellar and disc properties}

The $[\mathrm{OI}] 6300 \AA$ line is detected in 101 out of the 131 sources of our sample (i.e. $77 \%$ rate 

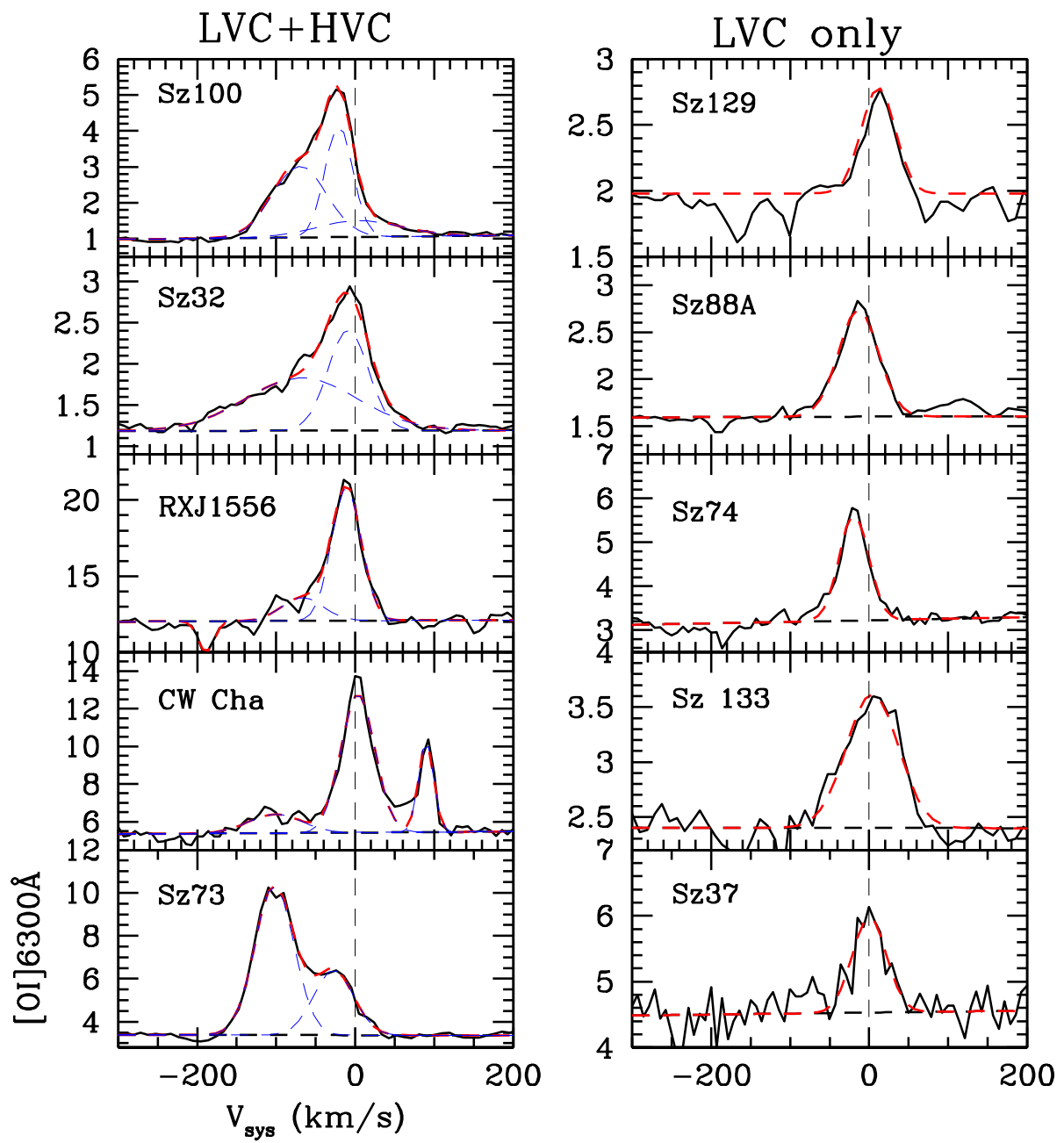

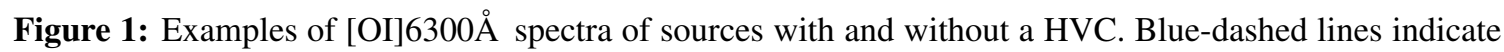
the gaussian profiles used to fit the different components, and the red-dashed line is the final best fit (From [4]).

of detection). In all detections the LVC is identified, while the HVC is observed in 39/131 objects (i.e. $30 \%$ rate of detection). In the majority of the sources the $L_{[O I] \mathrm{HVC}}$ is typically fainter than the $L_{[O I] \text { LVC }}$ by a factor of two/three, which partially explains the higher number of LVC detections with respect to the HVC. However sensitivity alone is not the only reason for a difference in detection rate between the LVC and $\mathrm{HVC}$, as the signal-to-noise ratio in the LVC line is often larger than 10 . We also estimate that orientation effects could misclassify emission at high velocity with a LVC in about $20 \%$ of sources (see discussion in [4]). The HVC detection rate does not depend on stellar luminosity, while a slightly larger detection rate is observed in sources with higher accretion luminosity (39\% in sources with $\left.\log \left(L_{\mathrm{acc}} / \mathrm{L}_{\odot}\right)>-3\right)$. 


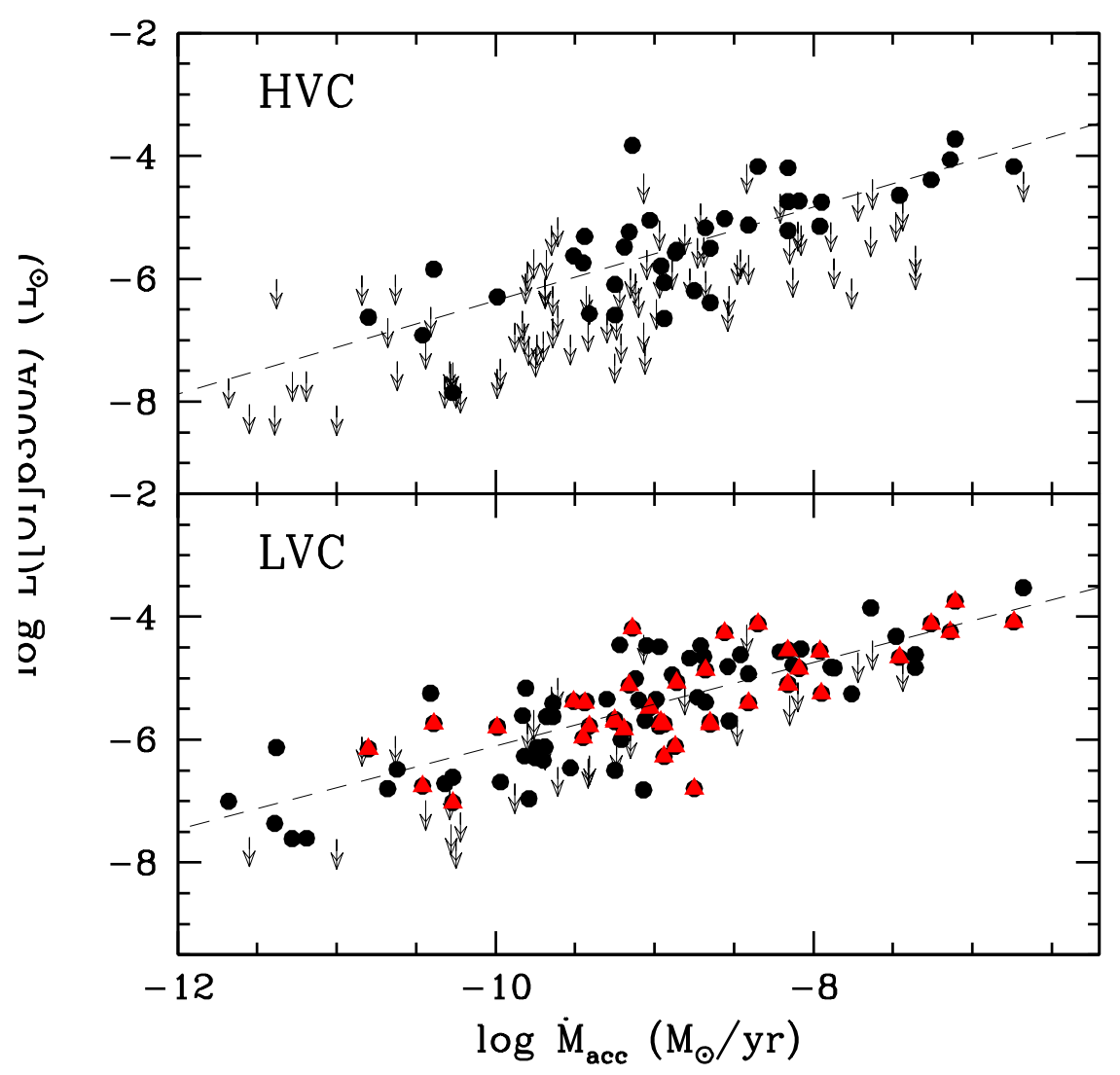

Figure 2: The [OI]6300 $\AA$ line luminosity of the LVC (bottom) and HVC (top) is plotted as a function of the mass accretion rate. Arrows refer to $3 \sigma$ upper limits. The dashed line indicates the linear regression. Red symbols in the LVC bottom plot indicate sources where also the HVC is detected. (Adapted from [4])

The $[\mathrm{OI}] 6300 \AA$ line luminosity has been correlated with the stellar and accretion parameters for both the LVC and HVC. The line luminosity correlates well with all the considered parameters in both LVC and HVC, with a Pearson correlation coefficient $r$ always between 0.7 and 0.8 . Correlations with $L_{*}$ present the larger scatter and in general we find that both the LVC and HVC show a better correlation with $L_{a c c}$ than with all the other parameters. The best fit linear regressions for all the correlations can be found in [4], while in Fig. 2 we show the correlation between the [OI]6300A luminosity and the mass accretion rate $\dot{M}_{a c c}$. The $\log \left(L_{[O I] \mathrm{HVC}}\right)$ vs $\log \dot{M}_{a c c}$ correlation is particularly useful to indirectly estimate the mass accretion rate in moderately embedded sources driving jets, where the permitted lines or the LVC emission region might be subject to large extinctions or scattering (e.g. [4]).

\section{The $\dot{M}_{j e t} / \dot{M}_{a c c}$ ratio}

In the assumption that the $[\mathrm{OI}] 6300 \AA$ HVC traces the high velocity jet, its luminosity can be used to infer the jet mass flux rate $\left(\dot{M}_{j e t}\right)$ adopting a procedure similar to that applied in [2] . In particular, to estimate the mass of the flow from the line luminosity we have considered the 
[OI]6300A emissivities computed considering a 5 level model, and assuming $T_{e}=10000 \mathrm{~K}$ and $n_{e}=5 \times 10^{4} \mathrm{~cm}^{-3}$, which typically represent the values found in the region at the base of T Tauri jets, where the [OI] emission originates. The adopted values are also similar to those used by [2] to estimate the $\dot{M}_{j e t}$ of a sample of Taurus sources from their HVC [OI] emission. Hence, we can make a direct comparison between our derived mass flux rates and those estimated by [2].

In Figure 3 the derived mass flux rates are plotted as a function of the source mass accretion rates. In this figure we also plot the data points relative to the sample of sources in Taurus whose $\dot{M}_{\text {jet }}$ has been measured by [2] with a similar procedure as the one we adopt here. For these sources, we have estimated the $\dot{M}_{a c c}$ from the determination of the accretion luminosity given by [16], adopting the stellar masses and radii given in the same paper. The Taurus sources, which extend to higher mass accretion rates with respect to our sample, fall along the same trend as the rest of targets. The Figure shows that for most of the detections the $\dot{M}_{j e t} / \dot{M}_{a c c}$ ratio is between 0.01 and 0.5 , confirming, on a large statistical bases, previous results found on individual objects. We note a significant number of upper limits pointing to a very low efficiency of the jet mass loss rate, even for objects with high accretion rates. In particular, the average of the detections is at $\dot{M}_{j e t} / \dot{M}_{a c c}$ $=0.07$ but considering both detections and upper limits, we find that 57 sources, i.e. $44 \%$ of the entire sample, have $\dot{M}_{j e t} / \dot{M}_{a c c}<0.03$.

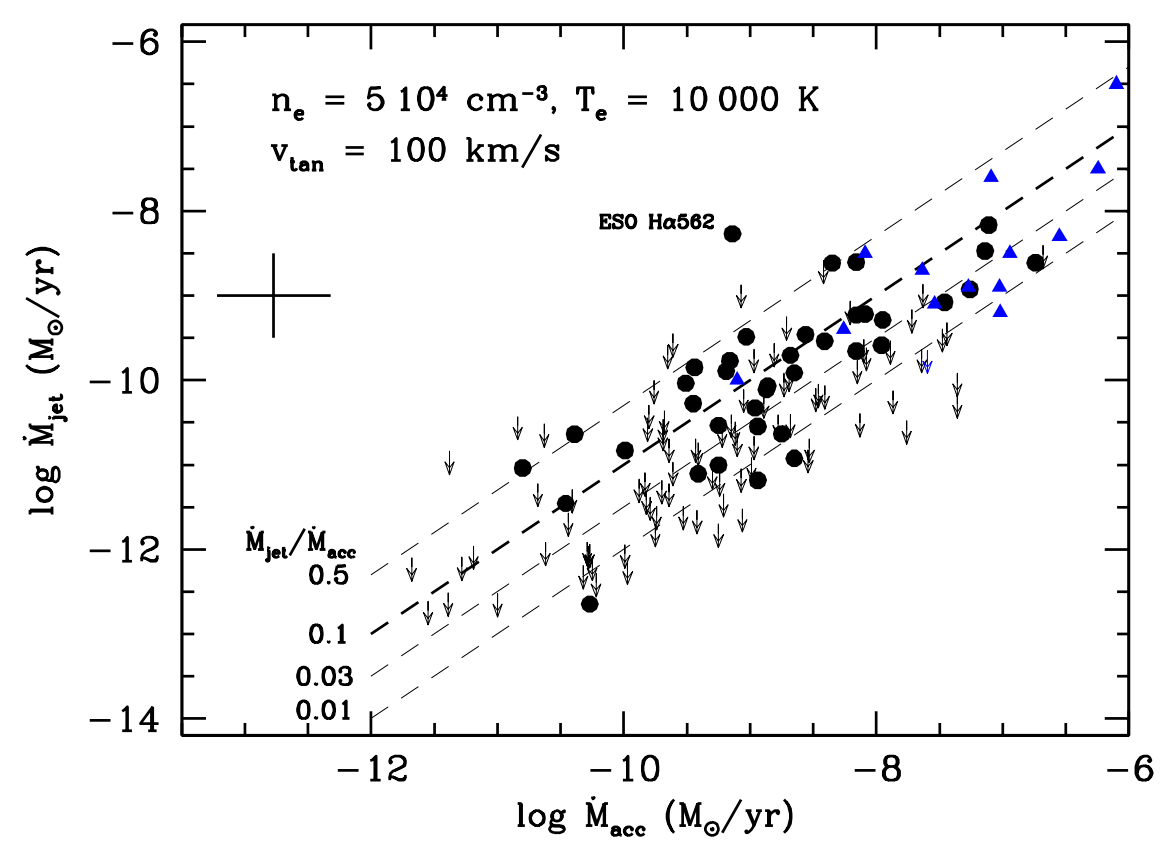

Figure 3: Mass accretion $\left(\dot{\mathrm{M}}_{a c c}\right)$ vs mass ejection $\left(\dot{\mathrm{M}}_{j e t}\right)$ rates. The $\dot{\mathrm{M}}_{\text {jet }}$ has been measured from the [OI] $6300 \AA$ luminosity assuming a jet tangential velocity of $100 \mathrm{~km} \mathrm{~s}^{-1}$ and gas temperature and density of $10000 \mathrm{~K}$ and $5 \times 10^{4} \mathrm{~cm}^{-3}$ respectively. Blue triangles indicate the values for the sample of Taurus sources (see text) (Adapted from [4])

The large scatter in the $\dot{M}_{j e t} / \dot{M}_{a c c}$ ratio is partially due to the uncertainty in the determination of the $\dot{M}_{j e t}$, and in particular on the assumed parameters. However, given the remarkable similarity 
in the excitation conditions of jets from sources with different masses and mass accretion rates, it is likely that the scatter, of almost two order of magnitude, reflects a real difference in the $\dot{M}_{j e t}$ efficiency among sources more than excitation conditions very different from what assumed here.

In conclusion, the analysis of $\dot{M}_{j e t} / \dot{M}_{a c c}$ ratio shows that there is a large spread of values for this ratio that does not depend on the mass of the driving source. From Fig. 3 we note, however, a tentative trend for sources with accretion rates $>10^{-8} \mathrm{M}_{\odot} \mathrm{yr}^{-1}$ to have on average a lower ratio. This trend needs to be confirmed/rejected on the bases of observations on a more complete sample of sources with high mass accretion rates.

\section{Conclusions}

We have summarised the results of a statistical work performed on the [OI]6300 sample of 131 young stars with discs in the Lupus, Chamaeleon and $\sigma$ Orionis star forming regions, having mass accretion rates spanning from $10^{-12}$ to $10^{-7} \mathrm{M}_{\odot} \mathrm{yr}^{-1}$, observed with the $\mathrm{X}$-shooter instrument. The line has been deconvolved into a LVC, detected in $77 \%$ of the sources, and in a HVC, present in only $31 \%$ of the objects. The luminosity of the two line components correlates with different stellar and accretion parameters of the sources. The line luminosity correlates better (i.e. has a lower dispersion) with the accretion luminosity than with the stellar luminosity or stellar mass. We suggest that accretion is the main drivers for the line excitation and that MHD discwinds are at the origin of both components. Mass ejection rates $\left(\dot{M}_{j e t}\right)$ measured from the detected HVC [OI]6300 $\AA$ line luminosity span from $\sim 10^{-13}$ to $\sim 10^{-7} \mathrm{M}_{\odot} \mathrm{yr}^{-1}$. The corresponding $\dot{M}_{j e t} / \dot{M}_{a c c}$ ratio ranges from $\sim 0.01$ to $\sim 0.5$, with an average value of 0.07 . However, considering the upper limits on the HVC, we infer a $\dot{M}_{j e t} / \dot{M}_{a c c}$ ratio $<0.03$ in more than $40 \%$ of sources. We argue that most of these sources might lack the physical conditions needed for an efficient magneto-centrifugal acceleration in the star-disc interaction region. Systematic observations of populations of younger stars, i.e. class $0 / \mathrm{I}$, are needed to explore how the frequency and role of jets evolve during the pre-main sequence phase. This will be possible in the near future thanks to space facilities like JWST.

\section{References}

[1] Frank, A., Ray, T. P., Cabrit, S., et al. 2014, Protostars and Planets VI, 451

[2] Hartigan, P., Edwards, S., \& Ghandour, L. 1995, ApJ, 452, 736

[3] Natta, A., Testi, L., Alcalá, J. M., et al. 2014, A\&A, 569, A5

[4] Nisini, B., Antoniucci, S., Alcalá, J. M., et al. 2018, A\&A, 609, A87

[5] Simon, M. N., Pascucci, I., Edwards, S., et al. 2016, ApJ, 831, 169

[6] Casse, F., \& Ferreira, J. 2000, A\&A, 353, 1115

[7] Ercolano, B., \& Owen, J. E. 2010, MNRAS, 406, 1553

[8] Alcalá, J. M., Manara, C. F., Natta, A., et al. 2017, A\&A, 600, A20

[9] Manara, C. F., Testi, L., Herczeg, G. J., et al. 2017, A\&A, 604, A127

[10] Alcalá, J. M., Natta, A., Manara, C. F., et al. 2014, A\&A, 561, A2 
[11] Rigliaco, E., Natta, A., Testi, L., et al. 2012, A\&A, 548, A56

[12] Espaillat, C., Muzerolle, J., Najita, J., et al. 2014, Protostars and Planets VI, 497

[13] Tazzari, M., Testi, L., Natta, A., et al. 2017, A\&A, 606, A88

[14] Appenzeller, I., \& Bertout, C. 2013, A\&A, 558, A83

[15] Caratti o Garatti, A., Eislöffel, J., Froebrich, D., et al. 2009, ApJ, 502, 579

[16] Rigliaco, E., Pascucci, I., Gorti, U., Edwards, S., \& Hollenbach, D. 2013, ApJ, 772, 60 